\title{
The temporal relationship between recall and subjective organization
}

\author{
C. RICHARD PUFF and DEBORAH A. VAN SLYKE \\ Franklin \& Marshall College, Lancaster, Pennsylvania
}

\begin{abstract}
Subjects were presented with a list of unrelated words in a multitrial free-recall paradigm. Items were correctly recalled significantly earlier in the sequence of trials than they were first entered into units of intertrial repetition (ITR). These findings corroborate Carterette and Coleman's (1963) conclusion and imply that subjective organization has little to do with making items initially accessible for recall. However, other findings suggest that subjective organization may be important in making items more reliably accessible. Additional analyses showed that, unlike "forward" ITR units, "reverse" ITR units remained significantly below chance across all trials. Implications of this for the use of the bidirectional ITR measure are discussed.
\end{abstract}

When people are asked to repeatedly recall a list of operationally unrelated words, they tend to remember the words in an increasingly fixed order. This phenomenon, referred to as subjective organization, was introduced by Endel Tulving (1962b). Tulving described a measure called SO, demonstrated the phenomenon, and began the exploration of its relationship to the amount of material that can be recalled. Bousfield, Puff, and Cowan (1964) developed a slightly different measure, called the intertrial repetition (ITR) score, and replicated some of Tulving's basic findings. Since then, a number of investigators have explored the parameters of the phenomenon, debated the virtues and limitations of several different measures, and, perhaps most importantly, searched for ways to demonstrate that this organization plays a causal role in mediating the process of recall (see Pellegrino \& Ingram, 1979, and Sternberg \& Tulving, 1977, for partial reviews of this research).

There seems to be a general acceptance of the idea that subjective organization is causally involved in recall. Sternberg and Tulving (1977), for instance, used the magnitude of the relationship between the amount of recall and values of various subjective organization measures as the means to evaluate the validity of those measures. However, despite the effort devoted to the issue, there still is no conclusive evidence of a direct causal relationship between recall and subjective organization as assessed by the SO and ITR measures. Even Tulving's (1962a) inferentially elegant direct-manipulation approach was inconclusive (e.g., see Puff, 1970). Consequently, the present study reflects the belief that the role of subjective organization, as defined by these original measures, remains an issue worthy of further critical evaluation.

This research was supported by a faculty research grant from Franklin \& Marshall College. Reprints may be obtained from C. R. Puff, Whitely Psychology Laboratories, Franklin \& Marshall College, P.O. Box 3003, Lancaster, PA 17604.
In one of the earliest evaluative studies, Carterette and Coleman (1963) proposed that subjective organization could not be causally involved in recall because of the temporal relationship between changes in the recall and SO measures. More specifically, they found that major increases in SO followed major increases in recall. Although it dealt with a potentially informative relationship, Carterette and Coleman's proposal apparently never received further examination. Because other lines of research have not yet provided conclusive evidence of a dependent relationship between subjective organization and recall, we thought it might be worthwhile to take another look at the temporal relationship between them.

The present approach differed somewhat from Carterette and Coleman's (1963). Here, the focus was on the temporal relationship between when (in terms of trial numbers) individual words were correctly recalled and when they entered into subjective organization units. The assumption was that if recall is critically dependent upon organization, then recall and organization must occur in close temporal contiguity. Because words have to be recalled to define the occurrence of this type of organization, the organization cannot be found to come first. However, organization and recall can occur simultaneously, and organization certainly should not follow recall by any significant interval.

A secondary focus on the comparative usefulness of measuring unidirectional and bidirectional units of subjective organization developed during the course of the study. Tulving's (1962b) original definition was based on unidirectional (forward) units, but in more recent years the measurement of bidirectional units has been preferred (e.g., see Sternberg \& Tulving, 1977). Sternberg and Tulving performed a careful analysis and concluded that, on the basis of standard psychometric criteria such as reliability and validity, the bidirectional measure was preferable. The bidirectional measure, however, is a combination of forward and reverse ITR units, and the nature of 
reverse units, to the best of our knowledge, has never been closely examined. Consequently, the present study included some exploration of the nature of reverse ITR units and of the comparative usefulness of unidirectional and bidirectional measures.

\section{METHOD}

\section{Materials}

A list of 16 words was presented with a slide projector and an automatic timing device. The words in the list were unrelated in the sense that, according to the Bousfield, Cohen, Whitmarsh, and Kincaid (1961) norms, they did not elicit each other directly in free association. All the words were common three-to-seven-letter nouns that began with different letters. Sixteen orders of presentation were used. Each word appeared once in each serial position across the 16 orders and was preceded and followed by each other word exactly one time. The orders of presentation were used in a sequence that was randomized for each subject. If a subject required more than 16 trials, the same randomizations were repeated.

\section{Procedure}

The subjects took part in the experiment individually. They were given instructions for multitrial free recall; these instructions stressed that they could recall the words in any order that seemed easy or natural. The words were presented at a rate of one item every $2.5 \mathrm{sec}$, and $90 \mathrm{sec}$ were allowed for each recall. All subjects received a minimum of 16 trials. If a performance criterion of two consecutive errorless recalls had not been reached by that time, additional trials were run until that criterion had been achieved or until a period of $1 \mathrm{~h}$ had elapsed.

\section{Subjects}

Twenty-four male introductory psychology students voluntarily participated in the study. Four of these subjects failed to reach the criterion of two consecutive errorless recalls within the 1-h period. Therefore, the results are based on the data from the 20 subjects who did reach the criterion.

\section{RESULTS}

The recall protocols were scored to provide several measures of recall and subjective organization. The recall measures were the number of the trial on which each item was correctly recalled for the first time and the trial on which consistent errorless recall began. Consistent recall was scored whenever an unbroken string of recalls began; this included the last trial if an item was recalled correctly then and had been missed previously. In those occasional instances in which a subject never consistently recalled an item, the subject's mean score was simply based on one fewer data point.

The subjective organization measures were the number of the trial (if any) on which each word first entered into a unit of ITR and the trial on which it entered into a consistent ITR unit, that is, one that was repeated throughout the remainder of the trials. The unbroken sequence of ITR units was said to have occurred on the last trial if it had not occurred before that and if the item had entered into an ITR unit then. Three types of ITR units, referred to here as forward, reverse, and bidirectional units, were scored. To understand the terminology, suppose that word A was followed by word B on some trial. If these same two words were recalled on the next trial in the same A-B order, a forward ITR unit was scored; if they were recalled in the reverse (B-A) order, a reverse ITR unit was scored; and if they were recalled in either order (A-B or B-A), a bidirectional ITR unit was scored. The three types of units were clearly not independent.

When there were duplicate recalls of the same item within a single protocol, any ITR units formed by either instance of the item were counted. This gave a slight bias toward the occurrence of ITR units early in the sequence of trials. In addition, before comparing the temporal occurrences of recall and subjective organization, an adjustment was made to the organization scores by subtracting a value of one trial from all of them. The traditional ITR measure scores a unit on the trial on which its formation is verified, that is, on trial $t+1$, the trial on which it is repeated. However, the logic of the measure is not changed by considering units to have the temporal locus of the trial on which they were formed, that is, on trial $t$. Because the use of the traditional procedure here would guarantee that there would be a one-trial lag between recall and the organization measure, this built-in bias was removed by subtracting one trial from the "trial of occurrence" scores for all of the organization measures.

The means and standard deviations of the various measures are shown in Table 1. It can be seen that there is a general pattern such that, for both first and consistent occurrences, recall came before entry into a bidirectional ITR unit, which came before entry into a forward ITR unit. Planned-comparison $t$ tests for repeated measures were performed between the recall and organization measures; the results are shown in Table 2 . The first recall of an item (at a mean of 1.96 trials) took place a significant number of trials before the item entered into any of the organization measures. Entry into the first occurrence of a bidirectional ITR unit (at 4.97 trials) was the next closest event, but was still 3 trials later. The first inclusion of an item in a forward ITR unit occurred at

Table 1

Means and Standard Deviations (SDs) of the Trials on Which the First and Consistent Occurrences of Recall, Forward ITRs, and Bidirectional ITRs Took Place

\begin{tabular}{|c|c|c|c|c|c|c|}
\hline \multirow[b]{2}{*}{ Occurrence } & \multicolumn{2}{|c|}{$\begin{array}{c}\text { Correct } \\
\text { Recall }\end{array}$} & \multicolumn{2}{|c|}{$\begin{array}{l}\text { Forward } \\
\text { ITR Unit }\end{array}$} & \multicolumn{2}{|c|}{$\begin{array}{l}\text { Bidirectional } \\
\text { ITR Unit }\end{array}$} \\
\hline & Mean & SD & Mean & SD & Mean & SD \\
\hline First & 1.96 & .55 & 6.66 & 1.88 & 4.97 & 1.37 \\
\hline Consistent & 8.64 & 3.72 & 15.09 & 3.44 & 13.38 & 3.66 \\
\hline
\end{tabular}

Note-One trial was subtracted from the ITR measures.

Table 2

Values of $\mathbf{t}$ Tests Comparing Recall and Organization Measures

\begin{tabular}{lrrrrr}
\hline & \multicolumn{2}{c}{ Forward ITR Units } & & \multicolumn{2}{c}{ Bidirectional ITR Units } \\
\cline { 2 - 3 } \cline { 5 - 6 } Recall & First & Consistent & & First & Consistent \\
\hline First & -11.48 & -17.18 & & -6.71 & -13.94 \\
Consistent & 2.78 & -9.43 & & 3.66 & -7.52 \\
\hline
\end{tabular}

Note-Negative $t$ values indicated that recall came before organization. With $d f=19$, all $p$ values were less than .05 . 
Table 3

Mean Recall and Subjective Organization Scores Across Trials

\begin{tabular}{|c|c|c|c|c|c|c|}
\hline \multirow[b]{2}{*}{ Trial } & \multirow[b]{2}{*}{ Recall } & \multicolumn{3}{|c|}{ Unidirectional ITRs } & \multicolumn{2}{|c|}{ Bidirectional ITRs } \\
\hline & & Forward & Reverse & Expected & Observed & Expected \\
\hline 2 & 10.90 & 1.00 & .65 & .70 & 1.65 & 1.39 \\
\hline 3 & 12.20 & 1.00 & 1.00 & 1.10 & 2.00 & 2.20 \\
\hline 4 & 12.95 & 1.30 & .75 & 1.18 & 2.05 & 2.31 \\
\hline 5 & 12.75 & 1.05 & .75 & 1.24 & 1.80 & 2.48 \\
\hline 6 & 12.65 & 1.30 & .75 & 1.24 & 2.05 & 2.48 \\
\hline 7 & 13.80 & 1.15 & 1.35 & 1.32 & 2.50 & 2.65 \\
\hline 8 & 13.55 & 1.45 & 1.20 & 1.44 & 2.65 & 2.88 \\
\hline 9 & 13.75 & 2.10 & 1.25 & 1.40 & 3.35 & 2.79 \\
\hline 10 & 14.55 & 3.20 & 1.30 & 1.45 & 4.50 & 2.91 \\
\hline 11 & 14.70 & 3.70 & .95 & 1.58 & 4.65 & 3.15 \\
\hline 12 & 15.35 & 4.20 & 1.15 & 1.68 & 5.35 & 3.35 \\
\hline 13 & 14.25 & 3.70 & 1.15 & 1.69 & 4.85 & 3.37 \\
\hline 14 & 15.10 & 3.60 & 1.75 & 1.57 & 5.30 & 3.15 \\
\hline
\end{tabular}

6.66 trials. Entry of an item into more reliable ITR units stretched out to 13.38 trials for a consistent bidirectional unit and 15.09 trials for a consistent forward unit.

The onset of consistent correct recall (at 8.64 trials) took place significantly later in the sequence of trials than did an item's first entry into a bidirectional ITR unit (at 4.97 trials) or a forward ITR unit (at 6.6 trials). However, consistent recall took place significantly before an item entered into a consistent bidirectional ITR unit (at 13.38 trials) or a consistent forward ITR unit (at 15.09 trials).

Mean recall and organization scores on each trial are presented in Table 3. Observed values of forward, reverse, and bidirectional units were compared against chance expected values in a repeated measures design with alpha set at .05 in all cases. The expected values were calculated by Pellegrino's (1971) method. For the forward measure, the observed values were not different from the expected values in the early part of the trial sequence, but they became significantly greater in the latter part of the sequence. The interaction was significant $[\mathrm{F}(14,266)$ $=9.57, \mathrm{MSe}=2.14 \mathrm{]}$. The number of reverse ITR units was significantly below chance overall $[F(1,19)=12.36$, $\mathrm{MSe}=1.02]$ and remained uniformly so over trials $[\mathrm{F}(14,266)=.89, \mathrm{MSe}=.65]$. The bidirectional units showed the same pattern as the forward units. A big rise over trials $[\mathrm{F}(14,266)=21.15, \mathrm{MSe}=2.54]$ brought the function for the observed values above that for the expected values in the second half of the trial sequence $[\mathrm{F}(14,266)=10.36, \mathrm{MSe}=1.84]$.

\section{DISCUSSION}

Our results show that, on the average, items are initially recalled significantly earlier in the sequence of trials than they are initially entered into ITR units. In other words, subjective organization, operationally defined in this specific way, does not occur until significantly after an item can be recalled. This implies that entry of an item into a subjective organization unit does not play a causal role in making that item initially accessible for recall. In this sense, our results corroborate Carterette and Coleman's (1963) conclusion.

There are, however, still other ways in which subjective organization may be causally involved in recall. One possibility is that the or- ganization of items A and B as a unit makes room, within a limited immediate memory capacity, for the recall of item $\mathrm{C}$ before that item is itself organized in some way. This is a logical possibility, but it is difficult to see how to assess it critically. The growth of ITR units over trials is consistent with this notion, inasmuch as a larger number of ITR units should allow more items to be accommodated within a limited capacity. However, the results show, much as Carterette and Coleman (1963) argued before, that most of the growth in ITR units took place after the subject could recall about 14 of the 16 words. That is, the number of ITR units did not begin to rise above chance until Trials 9 and 10 , by which time the subjects were already recalling almost all of the words.

Another possibility is that the organization has something important to do with making items more reliably accessible. More specifically, the organization may help to combat intertrial forgetting; that is, it may help to prevent the failure to recall an item that has previously been correctly recalled. Informal inspection of multitrial data reveals that a substantial change in the order or recall (i.e., a reduction in ITRs) on some trial is frequently accompanied by a big drop in the level of recall on that same trial. It is also the case that the first entry into an ITR unit came significantly before consistent recall. On the other hand, however, consistent recall came significantly before consistent entry into the same ITR unit. Perhaps the consistent entry into the same ITR unit is delayed by some tendency to reorganize in order to achieve an efficient structure for combatting intertrial forgetting.

Overall, there is no strong evidence for any of these possible causal roles for subjective organization defined in this specific way. It is still possible that the pairwise measures reflect a critically important memory process, but it has been exceedingly difficult to demonstrate that convincingly (e.g., see Mandler, Worden, \& Graesser, 1974). At the present, in contrast to the pairwise measures, the more heuristically useful concept of subjective organization seems to be a less operationally exact one (Mandler, 1979) more akin to an overall schema or structure for the list.

The results also showed that, in contrast to the findings for the forward ITR units, the number of reverse ITR units never got above chance. In fact, the number of reverse units remained significantly below chance throughout the sequence of trials. There is a constraint arising from the nonindependence of the forward and reverse measures, but that constraint would not start to operate until after the list was organized to over $50 \%$ of the maximum possible number of forward ITR units. However, the level of organization peaked just short of that here. Furthermore, to constrain the number of reverse ITRs to the level observed here, the mean number of forward ITRs would have had to have been about 13-14. In other words, the subjects could have imposed just as many reverse ITR units as they did forward units. Therefore, it looks as if they simply opted for the use of a unidirectional form of ordering as argued in Tulving's (1962b) original paper.

The generality of these findings on reverse ITRs needis to be assessed, but they seem to suggest that the bidirectional measure contains a component that is nonlinearly related to the development of fixed sequential ordering (as defined by the forward ITR measure). It is, therefore, hard to see how any inferential advantage can be gained by using these bidirectional scores. On the other hand, there is apparent conflict between these observations and the finding that an item entered into a bidirectional ITR unit earlier than into a forward ITR unit, and that the entry into a bidirectional ITR unit occurred temporally closer to the time of correct recall. This time the explanation may lie in the nature of the measures. The addition of any (i.e., reverse) ITR units to the forward units increases the probability that the bidirectional measure will show organization earlier and thereby brings the time of organization closer to the time of correct recall. The addition of even a chance (or belowchance) number of units makes a difference because the number of forward units is so low in the early trials. This same phenomenon may be involved in Sternberg and Tulving's (1977) findings of greater reliability and validity of the bidirectional measure. For example, since recall is increasing rapidly in early trials, any measure that shows more organization at that point will be more closely related to recall. At any rate, it is argued here that the bidirectional measure defines a different phenomenon and is not a "better" measure of the original phenomenon of the tendency to develop a fixed order of recall. 


\section{REFERENCES}

Bousfield, W. A., Cohen, B. H., Whitmarsh, G. A., \& Kincaid, W. D. (1961). The Connecticut free associational norms (ONR Tech. Rep. No. 35). Storrs, CT: University of Connecticut.

Bousfield, W. A., Puff, C. R., \& Cowan, T. M. (1964). The development of constancies in sequential organization during free recall. Journal of Verbal Learning and Verbal Behavior, 3, 489-495.

Carterette, E. C., \& Coleman, E. A. (1963). Some comments on Tulving's subjective organization. Paper presented at the meeting of The Psychonomic Society, Los Angeles, CA.

MANDLER, G. (1979). Organization, memory, and mental structures. In C. R. Puff (Eds.), Memory organization and structure. New York: Academic Press.

Mandler, G., Worden, P. E., \& Graesser, A. C., II. (1974). Subjective disorganization: Search for the locus of list organization. Journal of Verbal Learning and Verbal Behavior, 13, 220-235.

Pellegrino, J. W. (1971). A general measure of organization in free recall for variable unit size and internal sequential consistency. Behavior Research Methods \& Instrumentation, 3, 241-246.

Pellegrino, J. W., \& Ingram, A. L. (1979). Processes, products, and measures of memory organization. In C. R. Puff (Ed.), Memory organization and structure. New York: Academic Press.

Puff, C. R. (1970). Free recall with instructional manipulation of sequential ordering of output. Journal of Experimental Psychology, 84, $540-542$.

Sternberg, R. J., \& Tulving, E. (1977). The measurement of subjective organization in free recall. Psychological Bulletin, 84, 539-556.

Tulving, E. (1962a). The effect of alphabetical organization on memorizing unrelated words. Canadian Journal of Psychology, 16, 181-191.

TulviNG, E. (1962b). Subjective organization in free recall of "unrelated" words. Psychological Review, 69, 344-354.

(Manuscript received for publication August 27, 1984.) 